\title{
I GIOVANI MIGRANTI NELLE SCUOLE ITALIANE: PERCORSI FORMATIVI, DISUGUAGLIANZE, RISORSE
}

\author{
Maddalena Colombo*
}

\begin{abstract}
Nel 2013 la presenza di minori stranieri in Italia ha raggiunto quote numeriche importanti, soprattutto per la presenza di seconde generazioni (44\%). Famiglie e giovani stranieri puntano sull'istruzione anche a compensazione di diritti sociali che sentono negati (ius sanguinis); i livelli formativi degli immigrati stanno aumentando gradatamente, ma si segnalano alcune criticità: a) la persistenza di un gap negativo tra i risultati scolastici degli alunni di nazionalità italiana e straniera; b) in certi istituti la maggiore visibilità delle diverse provenienze etniche in alcune aree sembra provocare disagi nelle interazioni tra autoctoni e immigrati; c) in alcune sezioni scolastiche ad elevata incidenza di stranieri, la concentrazione di problematiche sociali mette in allarme la concezione anti-discriminatoria e liberale della scuola. Tuttavia anche in tali sezioni ci sono risorse educative che non vanno sottovalutate.
\end{abstract}

Parole chiave: scuola italiana, alunni con cittadinanza non italiana, seconde generazioni, disparità di trattamento, sociologia dell'educazione.

Il cambiamento culturale che sta vivendo il sistema formativo italiano, con l'ingresso di nuove generazioni sempre più multietniche, viene osservato da più di due decenni dagli esperti di varie discipline che studiano l'educazione, prima fra tutte la sociologia dell'educazione, che si occupa di fornire delle chiavi di lettura dei processi per facilitare il governo di tale mutamento.

Tradizionalmente questa disciplina ha fatto ricorso al concetto di "disuguaglianza", quale tratto costitutivo delle moderne economie di

\footnotetext{
* Docente di sociologia nell'Università Cattolica del Sacro Cuore di Milano. Milano/Italia.
} 
mercato, che si ripercuote "a valle" sugli squilibri tra i cittadini nell'accesso alle opportunità sociali. L'istruzione, ossia la partecipazione scolastica ma anche il successo formativo, sarebbero strumenti indispensabili per una politica di redistribuzione delle risorse sociali. In questa chiave, I'arrivo di studenti stranieri e la formazione di classi multietniche vengono visti come fattori critici, che fanno emergere nuove disuguaglianze basate sull'appartenenza culturale (linguistica, etnica, religiosa, ecc.), sulla disparità di status giuridico (cittadini italiani, non italiani, quasi-italiani, neo-italiani, italo-qualcos'altro, ecc.) ed infine sulla disparità di status socio-economico (è noto che il livello occupazionale e il reddito medio dei cittadini stranieri sono mediamente inferiori a quelli degli autoctoni). Da qui, la necessità di individuare quali di questi fattori si ripercuotono a loro volta sulla disuguaglianza formativa, quella legata ai risultati scolastici, agli apprendimenti e alle chance di successo dei ragazzi, e di proporre soluzioni correttive, preventive o di compensazione.

Ma il processo di trasformazione delle scuole in senso multiculturale non segue necessariamente una prospettiva conflittualista, e gli attori coinvolti nella mixité scolaire mostrano talvolta una capacità di adattamento superiore ai modelli previsionali, mirando anche a superare spontaneamente le disparità in ingresso, pur senza l'aiuto di opportune politiche scolastiche redistributive. Tra i fattori di maggiore o minore impatto negativo delle migrazioni sulle strutture scolastiche ci sono gli aspetti socio-demografici (quanti stranieri si iscrivono, in rapporto a quanti italiani, e a quale età si inseriscono per la prima volta nella scuola italiana), culturali (la relativa preparazione dei docenti e delle famiglie residenti all'accoglienza; la socializzazione anticipata dei newcomers alla lingua e alla cultura italiana, ecc.) e infine temporali: qual è il grado di stabilizzazione dei migranti in un territorio ${ }^{1}$, da quanto tempo la comunità scolastica sta affrontando il cambiamento, quali eventi critici accadono nel processo di inserimento scolastico, l'esistenza di un intervento locale (a corto o medio raggio) in favore dell'integrazione dei migranti, ecc. Il tempo può giocare in modo ambivalente, come elemento di stabilizzazione, ma anche di cronicizzazione delle problematiche non risolte.

Ciò che la sociologia può offrire, dunque, per sostenere le politiche in queste fasi di delicata transizione, è un monitoraggio continuo di situazioni specifiche, processi in corso, dinamiche strutturali, impatti derivanti da eventi

1 E' ormai provato che il grado di integrazione dei migranti aumenta con I'anzianità migratoria. Cf. gli indici di integrazione in CESAREO, Vincenzo e BLANGIARDO, G. Carlo (a cura di). Indici di integrazione. Un'indagine empirica sulla realtà migratoria italiana. 
imprevisti, ecc., fino a delineare un quadro tanto delle problematiche quanto delle risorse che scaturiscono dalla situazione. Per questo mi propongo di segnalare, in relazione al tema delle seconde generazioni a scuola, quattro dati di fatto e una possibile risorsa verso un'evoluzione positiva delle questioni in campo.

1) Nel 2013 la presenza di minori stranieri in Italia ha raggiunto quote numeriche importanti ( 1 milione di residenti, tra i quali 750 mila iscritti nei percorsi di istruzione e 180mila nei percorsi di formazione professionale ${ }^{2}$ ) e un peso "relativo" significativo (rappresentano il 9,7\% dei minori e l' $8,8 \%$ della popolazione scolastica) ${ }^{3}$, dati che prefigurano lo scenario multiculturale dei prossimi anni. Ma ora che la crescita dei flussi migratori, a causa della crisi economica, sta lentamente riducendosi, è forse opportuno dare spazio a qualche riflessione su tempi e modalità di una più efficace compartecipazione dei giovani immigrati e dei figli di immigrati nella vita del Paese. E' I'Istat, nel Bilancio demografico 2012, a segnalare che il tasso migratorio con I'estero, pur presentando valori ancora positivi con un contributo netto annuale di 280 mila unità ed un'incidenza complessiva degli stranieri del 7,4\% sul totale della popolazione residente ${ }^{4}$ lascia intendere un'inversione di passo: vi sono state meno iscrizioni anagrafiche rispetto agli anni scorsi (-35 mila nel 2011, - 73 mila nel 2010) e ben 106 mila cancellazioni, ossia persone che si sono trasferite definitivamente all'estero (di cui 1/3 stranieri) ${ }^{5}$.

2) Nello stesso tempo, la popolazione scolastica ed universitaria continua con gradualità ad assorbire studenti di origine straniera. Ciò indica che chi ha messo le radici nella società italiana sta investendo seriamente sulla cultura per ottenere diritti di accesso e una migliore qualità di vita in prospettiva. Come mostra il graf. 1, la curva dell'incidenza di studenti con cittadinanza non italiana a scuola e all'università negli ultimi 20 anni è illustrativa del contributo delle nuove generazioni di migranti al contesto formativo italiano.

2 Cf. MIUR - Fondazione Ismu. Approfondimenti e analisi; COLOMBO, Maddalena e SANTAGATI, Mariagrazia. Gli stranieri nel sistema della formazione professionale in Italia.

MIUR, Servizio Statistico. Gli alunni stranieri nel sistema scolastico italiano a.s. 2012/2013.

4 ISTAT. Bilancio demografico nazionale 2012. Popolazione residente, natalità, mortalità, migrazioni, famiglie e convivenze, p. 6.

5 Sta lentamente invertendosi la direzione di marcia delle popolazioni mobili, tra le quali occorrerà mettere in conto, nel giro di pochi anni, anche una emigrazione di giovani italiani verso i Paesi con maggiore dinamismo economico. Questo fenomeno porta a ridimensionare l'attuale percezione dell'Italia come "fortezza assediata". 


\section{GRAFICO 1 - Studenti stranieri iscritti a scuola e all'università Anni 1992/93-2008/09 (per 1000 iscritti) $^{6}$}

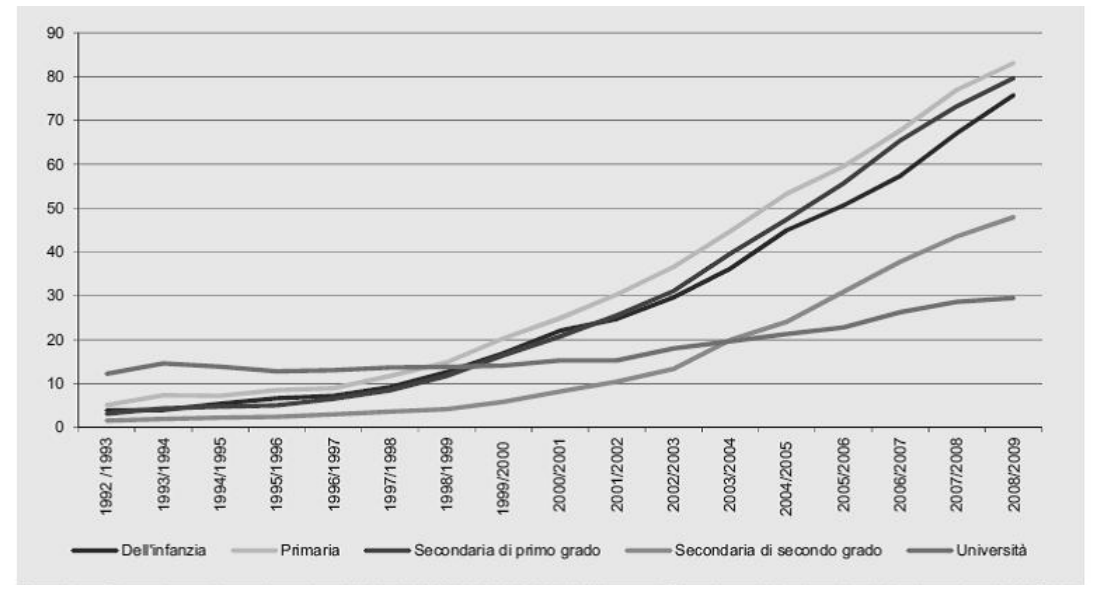

Fonte: ISTAT, rivelazione sulle scuole e università (anni 1992/1993-2000/2001; Ministero dell'istruzione, dell'università e della ricerca (anni 2001/2002-2008/2009)

Con I'indebolimento della crescita, sia in Italia che in Europa e nei Paesi dell'area occidentale, l'attenzione è sempre più puntata sulle generazioni "dopo" ${ }^{\prime \prime}$, coloro che sono nati nel Paese di emigrazione o ci sono arrivati prima di cominciare il percorso formativo e che molto probabilmente progettano di costruirsi lì una "carriera di vita". In Italia, malgrado la disciplina della cittadinanza giuridica si sia fermata sullo ius sanguinis e non riconosca loro uno statuto di parità con gli autoctoni, le scuole e tutti i servizi socio-educativi/ formativi si sono molto impegnati in questi anni per l'accoglienza dei figli degli immigrati e hanno investito energie per ottenere buoni risultati.

Le seconde generazioni rappresentano una compagine ormai consolidata nel panorama scolastico (il 47,2\% degli alunni con cittadinanza non italiana nell'a.s. 2012/13, +3\% nell'ultimo anno), che tende a crescere diversamente a seconda del grado (negli ultimi 4 anni è cresciuta del 9\% nelle scuole dell'infanzia, del $13 \%$ nelle primarie, del $10 \%$ nelle secondarie di 1 grado e del 3,4\% nelle secondarie di 2grado) ma che presto sarà maggioritaria - come del resto già lo è nella scuola dell'infanzia $80 \%$ e primaria $60 \%)^{8}$. Esse sono l'effetto della crescita delle nascite da genitori stranieri, la cui incidenza sul movimento demografico è passata da 4,8 ogni 100 nuovi nati nel 2000 a 14,9 ogni 100 nascite nel $2012^{9}$. E' sulla base di questo trend che Anci

\footnotetext{
${ }^{6}$ ISTAT. L'Italia in 150 anni, p. 351.

7 BARBAGLI, Marzio e SCHMOLL, Camille (a cura di). La generazione dopo.

${ }^{8}$ MIUR, Servizio Statistico. Gli alunni stranieri..., op. cit.

${ }^{9}$ ISTAT. Bilancio demografico nazionale 2012..., op. cit.
} 
e Cittalia ${ }^{10}$ hanno formulato la previsione di un raddoppio numerico entro il 2029: in quella data si conteranno quasi due milioni di minori stranieri che, in parallelo con il calo delle nascite degli autoctoni, arriveranno a rappresentare il 20,7\% dei minori residenti in Italia. Nel frattempo, se le richieste di cittadinanza proseguiranno al ritmo attuale (ipotesi di minima, perché è ragionevole sperare in una revisione in senso estensivo del diritto di cittadinanza nei prossimi anni), avremo 61.300 "nuovi" cittadini italiani, a parziale contropartita di tutti gli investimenti fin qui profusi.

3) $E^{\prime}$ importante infatti sottolineare che, se le seconde generazioni, una volta finiti gli studi in Italia, si unissero agli autoctoni nella ricerca di un lavoro adeguato all'estero, si avrebbe una significativa dispersione di risorse; la mancanza di accesso ai diritti legati alla cittadinanza giuridica potrebbe fare da incentivo alla loro fuoriuscita dalla società italiana. Al momento studi e ricerche sul campo confermano che famiglie e giovani stranieri puntano con convinzione sul Diritto-dovere di istruzione-formazione, per diverse ragioni: per possedere gli strumenti basilari di cittadinanza attiva (si pensi alle donne con competenza alfabetica scarsa o nulla che provengono da Paesi dove I'analfabetismo femminile è triplo di quello maschile ${ }^{11}$ ); per accedere alle credenziali di studio "compensando" gli altri diritti sociali formali che vengono loro negati; per conquistare chance di mobilità lavorativa e non sentirsi condannati a svolgere lavori "da immigrati"; infine per poter ottenere un riconoscimento in ambito sociale, qualcosa che ha a che fare con l'integrità della persona, il suo bisogno di approvazione da parte degli altri - perché senza riconoscimento c'è solo l'offesa e il disprezzo ${ }^{12}$.

Dal canto loro, le istituzioni scolastiche verificano quotidianamente come attraverso la scolarità dei figli e una buona amministrazione dei servizi, un'intera generazione di immigrati stia gradualmente passando "da residente a cittadino" con un aumento non solo dei livelli di integrazione delle famiglie ma anche delle competenze linguistiche minime, utili a far evolvere le comunità locali nelle quali essi vengono "incorporati"13. Infatti, tutti gli indicatori dell'integrazione utilizzati in ambito sia europeo sia italiano, comprendono la partecipazione scolastica (per i minori) o formativa (per gli adulti) quali elementi di stabilità e di miglioramento posizionale dei migranti ${ }^{14}$.

${ }^{10}$ GIOVANNETTI, Monia e NICOTRA, Veronica (a cura di). Da residenti a cittadini. II diritto di cittadinanza alla prova delle seconde generazioni, p. 120ss.

${ }^{11}$ Cf. WORLD ECONOMIC FORUM. Global Gender Gap Report 2012.

12 HONNETH, Alex. Riconoscimento e disprezzo. Sui fondamenti di un'etica post-tradizionale.

${ }_{13}$ PONZINI, Giuseppe (a cura di). Welfare e politiche per l'immigrazione, il decennio della svolta.

${ }^{14}$ CESAREO, BLANGIARDO, op. cit.; BIJL, Rob e VERWEIJ, Arjen (eds.). Measuring and monitoring immigrant integration in Europe; BOCCAGNI, Paolo e POLLINI, Gabriele. L'integrazione nello studio delle migrazioni. Teorie indicatori, ricerche. 
Ora il sistema scolastico ha nuove, importanti ragioni per investire sul capitale culturale dei migranti: innanzitutto perché la loro partecipazione assicura un ricambio demografico anche alle coorti scolastiche (si pensi a certe scuole rurali che sopravvivono grazie all'apporto dell'utenza migrante, o ai corsi serali, alle sezioni di istituti tecnico-professionali ecc.); poi perché la "fusione" dei bagagli linguistici, culturali e simbolici dei giovani, che avviene proprio nell'epoca della formazione adolescenziale, sembra che stia producendo nelle classi multietniche un mix interessante ai fini della costruzione di una mentalità "internazionalizzata", adatta al cosmopolitismo ${ }^{15}$.

4) Dando uno sguardo ai risultati fin qui ottenuti dal forte investimento degli ultimi anni, si può da un lato affermare che i livelli formativi degli immigrati stanno aumentando gradatamente, e che è in costante miglioramento l'accesso dei giovani stranieri alle informazioni che riguardano I'offerta formativa (non solo scolastica ma anche quella che proviene dai Centri di Formazione Professionale), fondamentali per effettuare scelte meno "canalizzate" ed imposte dalle contingenze ${ }^{16}$; vi è inoltre una diffusione più capillare dei dispositivi di sostegno ${ }^{17}$, una volta destinati solo alle regioni del Nord dove la la Formazione professionale è meglio attrezzata, oggi connessi con le azioni anti-dispersione al Sud di cui si avvantaggiano anche gli studenti stranieri.

Nondimeno si segnalano alcune criticità legate al rendimento scolastico, che le politiche di accoglienza non possono ignorare.

Innanzitutto la persistenza di un gap negativo tra i risultati scolastici degli alunni di nazionalità italiana e straniera ${ }^{18}$, con particolare allarme per la prima generazione. Anche il rischio di abbandono è ancora nettamente a sfavore degli studenti di origine straniera, sia nella scuola secondaria di 1 che di 2 grado con punte massime tra gli studenti della prima generazione ${ }^{19}$.

\footnotetext{
${ }_{15} \mathrm{Mi}$ riferisco agli esiti di alcune ricerche sulle seconde generazioni, che mettono in luce non solo I'impatto conflittuale con le strutture di opportunità ma anche l'apporto di nuove idee, ideologie, prospettive che derivano dalla condizione "sospesa" degli adolescenti di origine immigrata: BESOZZI, Elena; COLOMBO, Maddalena e SANTAGATI, Mariagrazia. Giovani stranieri, nuovi cittadini. Le strategie di una generazione ponte; COLOMBO, Enzo. Figli di migranti in Italia. Identificazioni, relazioni, pratiche; AMBROSINI, Maurizio (a cura di). Incontrarsi e riconoscersi. Socialità, identificazione, integrazione sociale tra i giovani di origine immigrata.

16 Infatti, come riportato dal notiziario Miur 2012, p. 13, la sperequazione tra stranieri e italiani nelle diverse filiere della scuola secondaria di 2 grado si è attenuata, soprattutto negli istituti tecnici (33,3\% italiani, $40,8 \%$ stranieri nati Italia; 38\% stranieri nati estero) e si sta recuperando il gap anche nei licei scientifici (a.s. 2011/12).

17 COLOMBO, Maddalena e SANTAGATI, Mariagrazia. Gli stranieri nel sistema della formazione professionale in Italia.

18 INVALSI - Ocse Pisa, Ocse Pisa 2012. Rapporto nazionale, p. 43.105.125.

${ }^{19}$ MIUR, Servizio Statistico. Focus "La dispersione scolastica".
} 
Una disuguaglianza reale che nasconde talvolta l'impreparazione delle scuole ad affrontare una didattica rivolta anche alle esigenze degli alunni stranieri, talvolta una mobilità eccessiva di questi studenti sul territorio, quando non una deprivazione socio-economica e culturale che è all'origine dell'insuccesso scolastico ${ }^{20}$.

In secondo luogo, si verifica in certi istituti una maggiore visibilità di alcune provenienze etniche, che sembra provocare disagi nelle interazioni tra autoctoni e immigrati (specie a livello degli adulti) anche quando tra bambini/ ragazzi non vi sono ragioni concrete per coltivare intolleranza reciproca e atteggiamenti xenofobi ${ }^{21}$. Vi è quindi il serio pericolo di una "etnicizzazione" delle situazioni problematiche.

Questo in realtà trova poco riscontro nelle analisi sull'incidenza della provenienza geografica sui destini/risultati scolastici degli allievi ${ }^{22}$ : se infatti si confrontano studenti che "vanno male a scuola" al netto delle differenze sociali (reddito, classe occupazionale, titolo di studio dei genitori) emerge che il differenziale tra stranieri e italiani si riduce notevolmente (meno 6 punti percentuali). Inoltre, alcune nazionalità di provenienza (come quelle dell'area sud-est asiatica, arabofona e africana sub-sahariana) che mostrano maggiore difficoltà tra i ragazzi di prima generazione, registrano invece nella seconda generazione un buon potenziale di miglioramento. Altre nazionalità, come quelle dell'area est-europea, si associano a buone performance scolastiche sia nella prima sia nella seconda generazione. Infine, si calcola che per i ragazzi che provengono dall'area latinoamericana l'influenza della variabile socio-economica, quindi la povertà materiale e il basso capitale socio-culturale delle famiglie, faccia da ostacolo al successo formativo più che per gli altri allievi di origine straniera.

Pertanto, se non ha fondamento attribuire ai ragazzi comportamenti o risultati scolastici "tipici" in base alla nazionalità o gruppo etnico di appartenenza, non ha senso neppure negare tali appartenenze né trascurare di considerare le condizioni sociali che i migranti vivono per comprendere i loro risultati scolastici e per intervenire con adeguate azioni di supporto. C'è

${ }^{20} C^{\prime}$ è tuttavia da segnalare che nelle prove di matematica il gap tra stranieri e italiani si sta riducendo - con riferimento alle seconde generazioni, tanto che nell'ultimo test Invalsi 2013 gli allievi stranieri nati in Italia hanno riportato il medesimo punteggio degli italiani in $2^{\circ}$ media (INVALSI - OCSE PISA, op. cit., p. 103).

${ }^{21}$ BESOZZI, Elena; COLOMBO, Maddalena e SANTAGATI, Mariagrazia. Misurare I'integrazione nelle classi multietniche; COLOMBO, SANTAGATI, op. cit.

${ }^{22}$ AZZOLINI, Davide e BARONE, Carlo. Do they progress or do they lag behind? Educational attainment of immigrants' children in Italy: The role played by generational status, country of origin and social class. 
infatti il forte rischio che si sottovaluti la possibilità di rimanere intrappolati nella spirale negativa: povertà à isolamento o auto isolamento nel contesto scolastico à rischio di etichettamento da parte dei pari à segregazione à aumento della deprivazione materiale e psicologica, che colpisce gli individui fragili nei contesti sociali complessi e selettivi. Se si accetta una formale "normalizzazione" della diversità delle condizioni di cui sono portatori gli studenti di origine immigrata ${ }^{23}$, il cosiddetto "circolo vizioso" può sempre rivelarsi operante nel contesto scolastico, anche malgrado l'accoglienza istituzionale; ciò avviene per effetto delle specifiche interazioni di gruppo ${ }^{24}$ e dinamiche interetniche ${ }^{25}$. Come mostrano i dati Eurostat (Graf. 2), la probabilità che la povertà si concentri tra i figli di chi è nato in un paese straniero, aumentando il differenziale coi figli dei nativi, introduce un fattore di disuguaglianza che la scuola, come principale istituzione perequativa per i minori, dovrebbe contrastare attraverso azioni di "discriminazione positiva".

\section{GRAFICO 2}

Minori a rischio povertà secondo il Paese di origine dei genitori (\% di popolazione), 2011 ${ }^{26}$

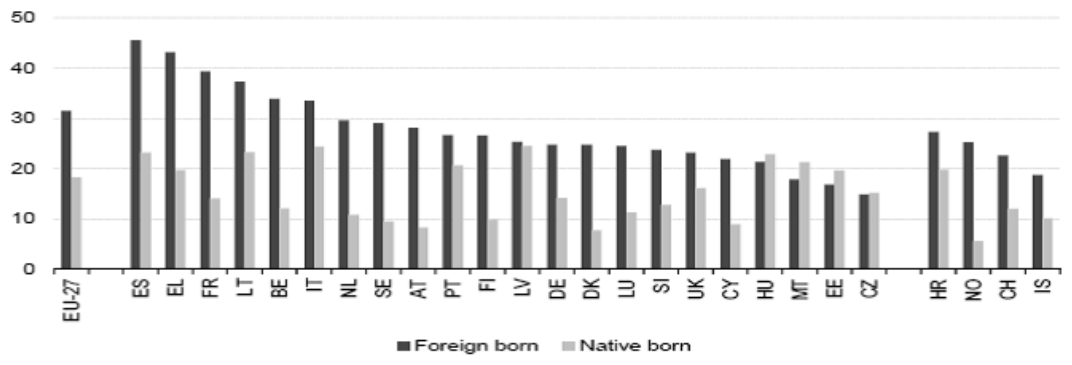

Note: EU-27 Eurostat estimation. Foreign born data is unreliable for BG, PL, RO and SK Source: Eurostat 2011.

Purtroppo il sistema scolastico italiano è tra quelli dove il retroterra socio-economico dei genitori influenza il risultato scolastico dei bambini più della media dei Paesi Ocse: il report Pisa 2009 ha calcolato che per ogni punto in meno nell'indice Socio-economico dello studente (ESCS), la differenza nei punteggi dei test in Italia è mediamente di 68, contro un differenziale medio

\footnotetext{
${ }^{23}$ BESOZZI, Elena. Introduzione: vecchie e nuove sfide per la scuola lombarda.

${ }^{24}$ SUAREZ-OROZCO, Carola e CARHILL, Avary. Andare avanti: la ricerca sui giovani immigrati e le loro famiglie.

25 SANTAGATI, Mariagrazia. Il contesto relazionale in classi ad alta concentrazionedi alunni stranieri; MARTINI, Elisa. Le seconde generazioni nella rete: un approccio di network allo studio delle relazioni sociali tra i banchi di scuola.

${ }^{26}$ EUROSTAT. Statistics in focus: Children were the age group at the highest risk of poverty or social exclusion in 2011, p. 5.
} 
dei Paesi Oecd di $62^{27}$. Molto dovrà dunque essere fatto nei prossimi anni per mitigare gli effetti negativi di questi meccanismi di disuguaglianza basati sulle condizioni sociali dei bambini, proprio perché svantaggiano tutti ed in particolare coloro che si trovano o si troveranno in situazione di fragilità materiale e famigliare, non solo a causa dell'origine migratoria: la scuola dovrà assumere in maniera rinnovata la sua tradizionale funzione di "grande equalizzatore" sociale.

Le nuove fragilità sono da individuare, oltre che fra gli studenti stranieri di prima generazione, anche fra gli allievi con rete famigliare ristretta o inesistente, con forte mobilità geografica, con scarsa "popolarità" nel gruppo dei pari. Le risorse che l'ente governativo potrà mettere a disposizione per l'integrazione dovranno andare prioritariamente alle scuole con poca disponibilità di supporti locali extrascolastici, agli insegnanti con nessuna formazione alle spalle per insegnare L2 (seconda lingua), ai dirigenti alle prese con processi di "sostituzione demografica" e con "reazioni della maggioranza" e difficile presa in carico da parte dell'ente locale.

5) L'ultimo punto di questo contributo vuole guardare avanti in un prospettiva di migliore utilizzo delle risorse già esistenti: tra queste mi permetto di segnalare la positiva combinazione delle diversità presenti nelle classi multietniche che, a dispetto di previsioni basate sui trend delle passate generazioni, non necessariamente porta agli effetti negativi temuti. $E^{\prime}$ quanto emerge dalla ricerca svolta in Lombardia dal Gruppo scuola dell'Osservatorio Orim $^{28}$, dove è stato possibile considerare l'influenza della percentuale di stranieri (il campione era formato da classi di scuola secondaria di 1 grado con più del $30 \%$ di stranieri iscritti) sulle relazioni tra compagni e sul loro successo scolastico. Indubbiamente la situazione di "malessere" degli studenti è risultata correlata con lo scarso rendimento degli allievi, ma si è provato che non è tanto l'incidenza stranieri/italiani che la provoca, quanto una composizione omogenea di genere (maschile) e di classe sociale (alunni autoctoni e immigrati provenienti da famiglie con basso indice di status, che sono molto frequenti nelle classi con alte percentuali di stranieri).

La ricerca ha misurato i livelli di integrazione degli alunni, sia immigrati che nativi: la percentuale di allievi stranieri in classe figura agli ultimi posti tra gli elementi predittori della riuscita scolastica (conta di più il livello di benessere, lo status dei genitori, I'essere femmine e nati in Italia) e non figura tra gli elementi predittori delle tensioni tra pari (conta di più il

\footnotetext{
${ }^{27}$ OECD. Equity and Quality in Education. Supporting disadvantaged students and schools, p. 108.

${ }^{28}$ BESOZZI, COLOMBO, SANTAGATI, Misurare l'integrazione..., op. cit.
} 
livello di benessere, l'essere maschi, il livello di relazionalità/fiducia interna alla classe, l'accettazione verso l'immigrazione), anche se le tensioni sono connesse alla cittadinanza non italiana dei soggetti che subiscono o assistono a prepotenze tra compagni. Inoltre, l'indice di accettazione dell'immigrazione è positivamente correlato alla situazione di classe: cioè aumenta con il crescere della percentuale di immigrati in classe, il che porta a sottolineare la valenza positiva del contatto quotidiano tra le diversità che "apre" le prospettive personali anche degli allievi italiani.

In sostanza occorre guardare con scrupolo, ma anche con ottimismo, alle classi multietniche, non solo come possibili luoghi di segregazione formativa (per evitarla, occorre prestare più attenzione a come vengono formate le classi, sul piano della mescolanza non solo delle origini etnico-linguistiche ma anche della eterogeneità di condizioni famigliari e di genere), ma anche come una nuova frontiera del cambiamento multiculturale delle scuole in Italia. In esse si trovano risorse particolari: si pensi al fatto che un numero più elevato di alunni stranieri in classe significa una maggiore concentrazione di spirito di adattamento, volontà di riuscita, "ottimismo" verso il futuro e rispetto delle regole di convivenza, ecc., tratti comuni a molti migranti tanto da costituire in taluni casi un modello di comportamento per i coetanei e per le loro stesse famiglie. Le "scuole plurali" si preparano ad affrontare e a vincere una nuova sfida, quella di dimostrare che gli investimenti fatti sulle nuove generazioni della mixité portano alla diffusione di valori democratici e pluralistici di cui il sistema educativo oggi ha segnatamente bisogno ${ }^{29}$.

\section{Bibliografia}

AMBROSINI, Maurizio (a cura di). Incontrarsi e riconoscersi. Socialità, identificazione, integrazione sociale tra i giovani di origine immigrata. Milano: Orim - Fondazione Ismu, 2011.

AZZOLINI, Davide; BARONE, Carlo. Do they progress or do they lag behind? Educational attainment of immigrants' children in Italy: The role played by generational status, country of origin and social class. Research in Social Stratification and Mobility, v. 31, 2013, p. 82-96.

BARBAGLI, Marzio; SCHMOLL, Camille (a cura di). La generazione dopo. Bologna: II Mulino, 2011.

BIJL, Rob; VERWEIJ, Arjen (eds.). Measuring and monitoring immigrant integration in Europe. The Hague: The Netherlands Institute for Social Research, Scp, 2012. BESOZZI, Elena. Introduzione: vecchie e nuove sfide per la scuola lombarda. In AA.VV. Rapporto 2013. Gli immigrati in Lombardia. Milano: Orim, 2014 (in corso di stampa).

${ }^{29}$ COLOMBO, Maddalena et alii (a cura di). Pluralism and diversity management in education. 
BESOZZI, Elena; COLOMBO, Maddalena; SANTAGATI, Mariagrazia. Giovani stranieri, nuovi cittadini. Le strategie di una generazione ponte. Milano: Franco Angeli, 2009. BESOZZI, Elena; COLOMBO, Maddalena; SANTAGATI, Mariagrazia. Misurare I'integrazione nelle classi multietniche. Rapporto regionale Orim 2012. Milano, 2013. BOCCAGNI, Paolo; POLLINI, Gabriele. L'integrazione nello studio delle migrazioni. Teorie indicatori, ricerche. Milano: Franco Angeli, 2012.

CESAREO, Vincenzo; BLANGIARDO, G. Carlo (a cura di). Indici di integrazione. Un'indagine empirica sulla realtà migratoria italiana. Milano: Franco Angeli, 2009.

COLOMBO, Enzo (a cura di). Figli di migranti in Italia. Identificazioni, relazioni, pratiche. Torino: Utet, 2010.

COLOMBO, Maddalena et alii (a cura di). Pluralism and diversity management in education. Italian Journal of Sociology of education, v. 5, n. 2, 2013 (co-editors: Brereton Bernadette, Kantzara Vasiliki, Sebastiao João, Van Houtte Miecke). Disponibile su: <http://www.ijse.eu/index.php/ijse/issue/view/16/showToc>.

COLOMBO, Maddalena; SANTAGATI, Mariagrazia. Gli stranieri nel sistema della formazione professionale in Italia. In FONDAZIONE MORESSA (a cura di). Terzo Rapporto sull'economia dell'immigrazione. Bologna: Il Mulino, 2013, p. 133-159. COLOMBO, Maddalena; SANTAGATI Mariagrazia. Nelle scuole plurali. Misure di integrazione degli alunni stranieri. Milano: Franco Angeli, (in corso di stampa), 2014.

EUROSTAT. Statistics in focus: Children were the age group at the highest risk of poverty or social exclusion in 2011. N. 4, 2013 (ed. Lopez-Vilaplana C.).

GIOVANNETTI, Monia; NICOTRA, Veronica (a cura di). Da residenti a cittadini. II diritto di cittadinanza alla prova delle seconde generazioni. Roma: Cittalia-Anci, 2012.

HONNETH, Axel. Riconoscimento e disprezzo. Sui fondamenti di un'etica posttradizionale. Soveria Mannelli: Rubbettino, 1993.

INVALSI - OCSE PISA. Ocse Pisa 2012. Rapporto nazionale. Roma, 2013. Disponibile su: <http://www.invalsi.it/invalsi/ri/pisa2012/rappnaz/Rapporto_NAZIONALE_ OCSE_PISA2012.pdf $>$.

ISTAT. L'Italia in 150 anni. Roma, 2011.

. Bilancio demografico nazionale 2012. Popolazione residente, natalità, mortalità, migrazioni, famiglie e convivenze. Roma, 25.6.2013.

MARTINI, Elisa. Le seconde generazioni nella rete: un approccio di network allo studio delle relazioni sociali tra i banchi di scuola. In BARBAGLI, Marzio; SCHMOLL, Camille (a cura di). La generazione dopo. Bologna: Il Mulino, 2011, p. 233-263.

MIUR. Gli alunni con cittadinanza non italiana nel sistema scolastico italiano, A.s. 2011/12. Roma, ottobre 2012.

. Servizio Statistico. Gli alunni stranieri nel sistema scolastico italiano a.s. 2012/2013. Roma, ottobre 2013.

. Servizio Statistico. Focus "La dispersione scolastica". Roma, giugno 2013.

MIUR-FONDAZIONE ISMU. Approfondimenti e analisi. Rapporto nazionale a.s. 2011/12 (a cura di Vinicio Ongini e Mariagrazia Santagati). Quaderni Ismu, n. 1, 2013. 
OECD. Equity and Quality in Education. Supporting disadvantaged students and schools. Paris: OECD Publishing, 2012.

PONZINI, Giuseppe (a cura di). Welfare e politiche per l'immigrazione, il decennio della svolta. Rapporto IRPPS CNR sullo Stato sociale in Italia 2012. Napoli: Liguori, 2012. SANTAGATI, Mariagrazia. II contesto relazionale in classi ad alta concentrazionedi alunni stranieri. In BESOZZI, Elena; COLOMBO, Maddalena; SANTAGATI, Mariagrazia. Misurare l'integrazione nelle classi multietniche. Rapporto regionale Orim 2012. Milano: OIM, 2013, p. 45-68.

SUAREZ-OROZCO, Carola; CARHILL, Avary. Andare avanti: la ricerca sui giovani immigrati e le loro famiglie. In BARBAGLI, Marzio; SCHMOLL, Camille (a cura di). La generazione dopo. Bologna: Il Mulino, 2011, p. 29-51.

WORLD ECONOMIC FORUM. Global Gender Gap Report 2012 (a cura di Zahidi Saadia). Berkeley, 2012.

\section{Abstract}

\section{Young migrants in italian schools: learning paths, inequality, resources}

In 2013, the presence of foreign minors in Italy reached significant figures, especially for the presence of second generations (44\%). Some families and young foreigners strive for social rights regarding education that they feel they are denied (ius sanguinis); educational levels of migrants are increasing gradually, but they indicate some critical issues: a) the persistence of a negative gap between the educational performance between students of Italian nationality and foreign nationality; b) in certain areas and institutions, increased visibility of different ethnic backgrounds seems to cause inconvenience in the interactions between natives and immigrants; c) in some educational sections with high levels of foreigners, the concentration of social problems threatens the anti-discrimination concept and liberal school. However, even in these sections there are educational resources that should not be overlooked.

Keywords: Italian school, pupils with non-Italian citizenship, second generations, disparity of treatment, sociology of education.

Articolo ricevuto il 20/01/2014. Accettato per la pubblicazione il 12/05/2014. Received for publication on January, 20 2014. Accepted for publication on May, 12 $2^{\text {th }}, 2014$. 